\title{
Acute myocardial infarction with left main thromboses in a young amphetamine addict
}

Kitigon Vichairuangthum

Division of Cardiology, Phatthalung-Thonburi Hospital Heart Centre, Phatthalung, Thailand

Submitted: 4 January 2020

Accepted: 25 April 2020

Arch Med Sci Atheroscler Dis 2020; 5: e45-e48

DOI: https://doi.org/10.5114/amsad.2020.95381

Copyright $\odot 2020$ Termedia \& Banach

Amphetamines are common in drug abuse, particularly in the young population. These sympathomimetic amines work by activating the sympathetic nervous system. As a result, the heart rate increases and the oxygen supply decreases, causing the oxygen demand of the heart to increase. However, the most common cardiovascular effects are tachycardia and hypertension. Acute myocardial infarction (AMI) is reported rarely after the use of amphetamines, especially in young patients [1]. We report a case of a healthy, young man who presented to our emergency department after oral consumption of amphetamines. In this case, amphetamine abuse caused myocardial ischaemia due to blood clot formation.

A 21-year-old university student was brought by his teacher to the emergency department with severe chest pain and palpitation for $2 \mathrm{~h}$. He was of average build with no history of similar symptoms, such as ischaemic heart disease, diabetes mellitus, hypertension, dyslipidaemia, or atrial fibrillation. He had no family history of similar symptoms or coronary artery disease. He was not on any medication. He had no allergies and no history of smoking or drinking alcohol. The patient used amphetamine tablets occasionally for recreational purpose and reported last taking an amphetamine tablet about $7 \mathrm{~h}$ before the presentation. At the time of arrival to the emergency room, his blood pressure was $95 / 60 \mathrm{~mm} \mathrm{Hg}$, heart rate was 130 beats per minute, respiratory was 18 breaths per minute, oral temperature was $37.5^{\circ} \mathrm{C}$, and oxygen saturation was $98 \%$ in room air. Generally, he appeared with agitation and severe chest discomfort. His ECG demonstrated atrial fibrillation with marked ST segment elevation at augmented unipolar right arm lead (aVR) and diffuse ST segment depression at the residual leads. Echocardiogram showed poor left ventricle ejection fraction (LV EF) 30\% with global hypokinesia and mild mitral regurgitation. Other laboratory assessment revealed evidence of myocardial injury, with positive troponin I. Coronary angiography showed a total occlusion of the left main (LM) artery (Figure 1) but normal right coronary artery (RCA) (Figure 2). Percutaneous coronary intervention was selected as the treatment strategy according to the patient's decision. An intra-aortic balloon pump (IABP) was inserted via the left groin.

The LM artery was engaged with a $7 \mathrm{Fr}$ Launcher EBU 3.5 guiding catheter (Medtronic, Minnesota, USA) via the right groin. The distal part of the LCX was wired with pilot 50 wires (Abbott Vascular, SantaClara, CA, USA). The LM clot was aspirated using an Export aspiration catheter (Medtronic, Minnesota, USA) and ballooned at the proximal part of the

\author{
Corresponding author: \\ Kitigon Vichairuangthum \\ Division of Cardiology \\ Phatthalung-Thonburi \\ Hospital Heart Centre \\ Phatthalung, Thailand \\ E-mail: neozz15@hotmail. \\ com
}




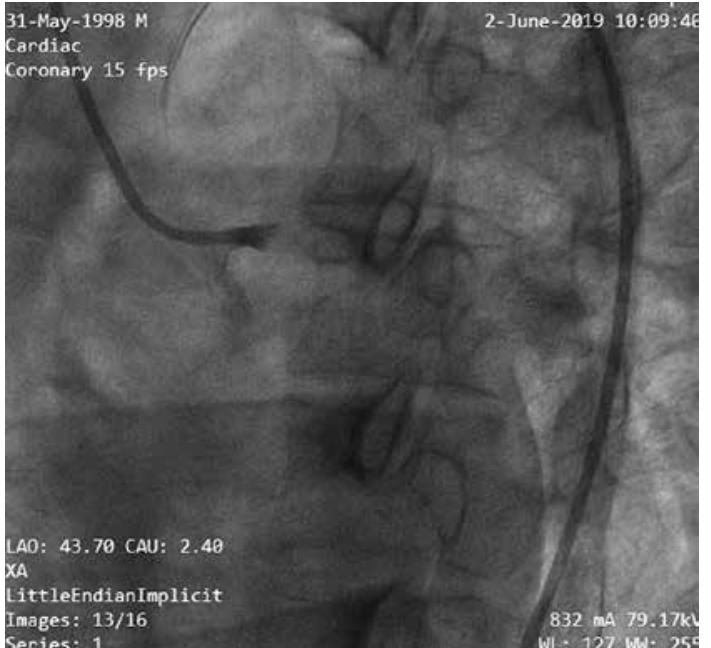

Figure 1. Initial coronary angiogram of left coronary artery (left caudal view)

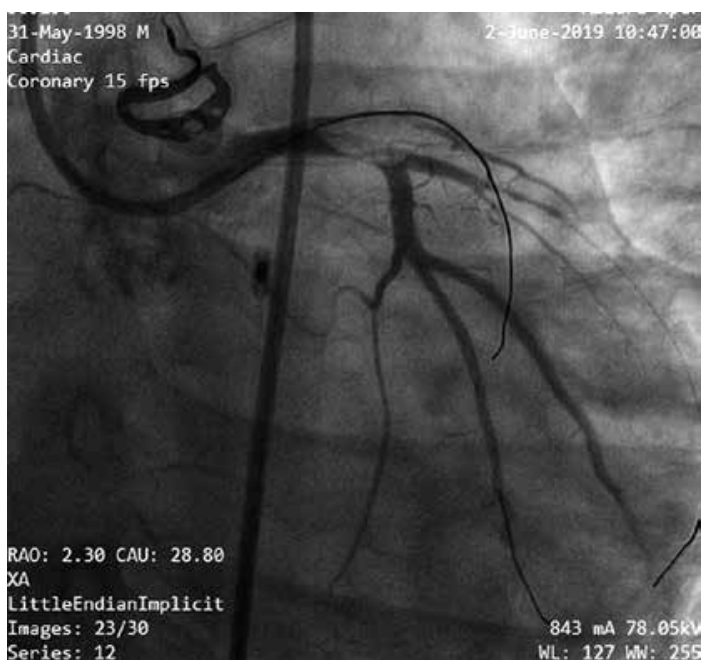

Figure 3. Residual clot at left main-left circumflex artery after clot was aspirated (right caudal view)

LCX with Minitrek (Abbott Vascular, SantaClara, CA, USA) $2.5 \times 15 \mathrm{~mm}$. The left anterior descending (LAD) artery was wired with a Miracle 6 wire (Asahi Intecc Co. Ltd, Aichi, Japan). There was a residual clot at the LM-LCX after multiple clot aspiration (Figure 3). The clot was re-aspirated (Figure 4) and the bifurcated distal LM was stented by Double Kissing Crush stenting technique (DK CRUSH), step by step: 1) balloon NC trek (Abbott Vascular, Santa Clara, CA, USA) $2.5 \times 15 \mathrm{~mm}$ in LM-LAD and Firebird 2 drug-eluting stent (DES) (MicroPort, Shanghai, China) was introduced into the LCX; 2) then the balloon in the LM-LAD was inflated to crush the deployed LCX stent; 3 ) after rewiring LCX proximally, first kissing was performed; 4) stenting LM-LAD with a $4.0 \times 13 \mathrm{~mm}$ Firebird 2 drug-eluting stent (DES); and 5) the final kissing balloon inflation was conducted after rewiring the LCX from the proximal LM-LAD stent cell. After

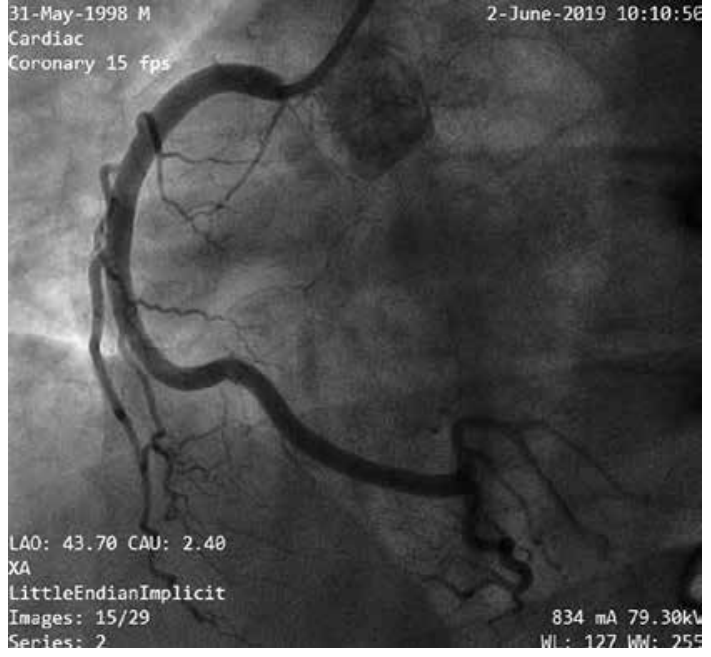

Figure 2. Initial coronary angiogram of right coronary artery (left caudal view)

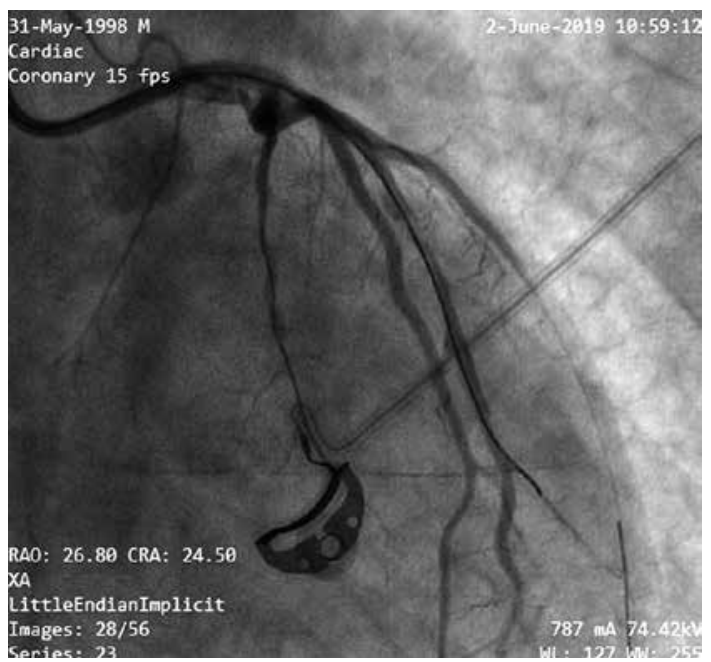

Figure 4. Angiogram before stent implantation (right cranial view)

stenting, a proximal optimisation technique was performed with NC balloon trek (Abbott Vascular, SantaClara, CA, USA) $4.0 \times 8 \mathrm{~mm}$ to improve the stent strut apposition. The result of the procedure was excellent with thrombolysis in myocardial infarction (TIMI) flow (TIMI-3) achieved in all branches (Figures 5, 6). No immediate complication occurred. A future plan and multidisciplinary team follow-up with psychiatric consultation was set before discharging the patient home. He was discharged from the hospital after one week with stable condition.

Amphetamines are potent sympathomimetic agents. The primary mechanism of action is blockage of the presynaptic reuptake of catecholamines and enhanced sympathetic stimulation; the most common cardiovascular side effects are a rise in pulse and blood pressure [2]. Other common complaints can be chest pain and shortness of breath. 


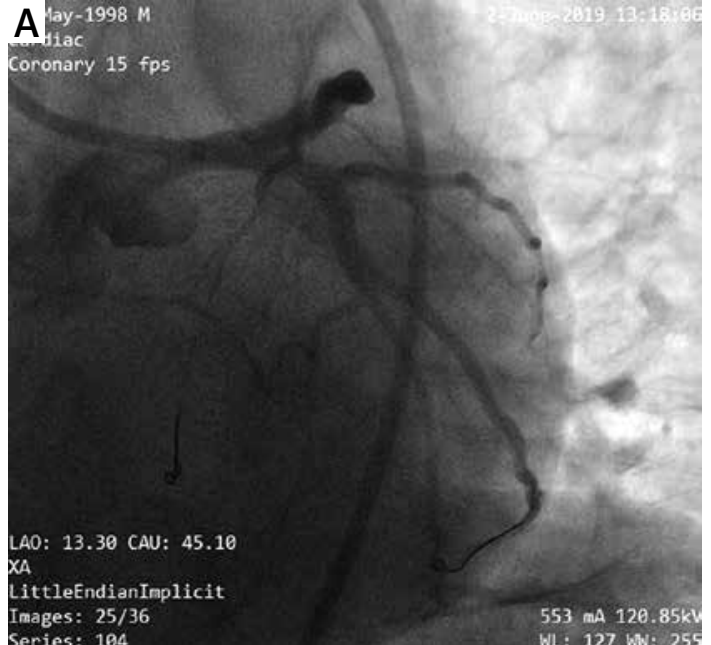

Figure 5. Good thrombolysis in myocardial infarction flow all branches after stenting (left caudal view)

The cardiovascular effects of amphetamines include a strong association with $\mathrm{AMI}[3,4]$, probably related to catecholamine surge, coronary vasospasm, and coronary thrombosis. Additionally, the chronic use of amphetamines is described to promote coronary atherosclerosis [5]. Amphetamines induce endothelial tissue factor expression and impair tissue factor pathway inhibitor. They also increase expression of plasminogen activator inhibitor-1 (PAl-1), a key fibrinolysis suppressant in human vascular endothelial cells $[3,6]$. Moreover, amphetamines are known to increase platelet aggregation and trigger atherosclerotic plaque rupture $[2,6]$. This patient's original severe left ventricular systolic dysfunction and generalised wall motion abnormality might be due to $\mathrm{AMI}$; however, amphetamine is reported to induce acute cardiomyopathy $[7,8]$. To our knowledge, there are no publications of amphetamine-induced acute myocardial infarction with total occlusion of the left main artery. By contrast, cases with abnormalities in the LAD artery [1, 7], right coronary artery (RCA) [9], or left circumflex artery (LCX) alone [4], due to amphetamine use, have been reported. Moreover, the mechanism of drug-induced $\mathrm{MI}$ is usually associated with coronary spasms $[5,10,11]$. The lack of vasospasms and the presence of thrombosis in our case (Figure 7) are unusual in literature.

Amphetamine-associated AMI may occur more commonly in the acute care setting if the rate of amphetamine abuse continues to increase. We find this case of amphetamine-induced AMI to be of particular interest for understanding the range of possible cardiovascular issues we may encounter in our emergency room, because early diagnosis and prompt treatment can be the key to successful management of this potentially fatal complication.

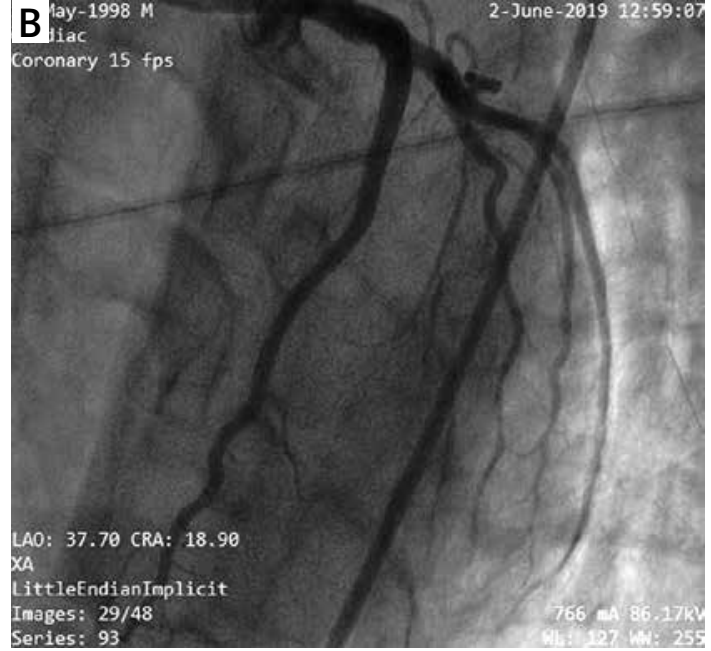

Figure 6. Good thrombolysis in myocardial infarction flow all branches after stenting (left cranial view)

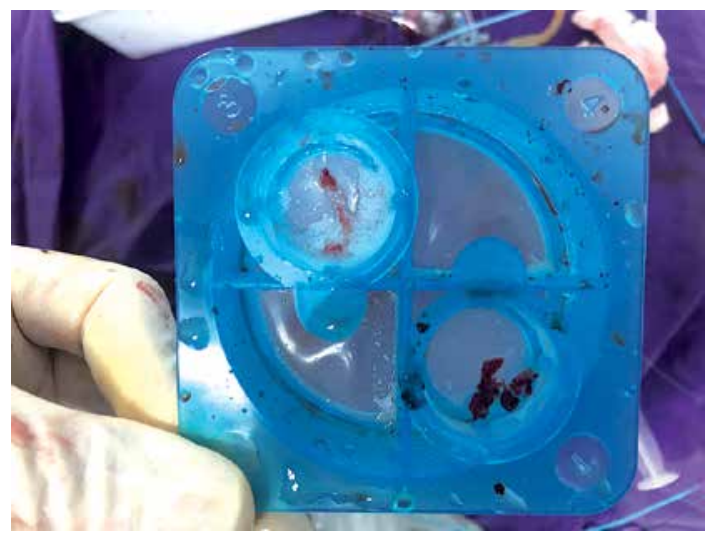

Figure 7. Clots retrieved from coronary artery

In conclusion, this case of amphetamine-induced AMI, with total LM thrombosis, is a good example for understanding the range of possible drug-induced cardiovascular manifestations and how to manage a difficult life-threatening situation. Despite young age, significant thrombus burden may be present. In patients with typical electrocardiogram (ECG) changes and elevated troponin levels, coronary angiography is indicated in this population.

\section{Conflict of interest}

The author declares no conflict of interest.

\section{Reference}

1. Khaheshi I, Mahjoob MP, Esmaeeli S, Eslami V, Haybar H. Simultaneous thrombosis of the left anterior descending artery and the right coronary artery in a 34-year-old crystal methamphetamine abuser. Korean Circ J 2015; 45: 158-60.

2. Albertson TE, Derlet RW, Van Hoozen BE. Methamphetamine and the expanding complications of amphetamines. West J Med 1999; 170: 214-9. 
3. Gebhard C, Breitenstein A, Akhmedov A, et al. Amphetamines inducetissue factor and impair tissue factor pathway inhibitor: role of dopamine receptor type 4. Eur Heart J 2010; 31: 1780-91.

4. Khattab E, Shujaa A. Amphetamine abuse and acute thrombosis of left circumflex coronary artery. Int J Case Rep Images 2013; 4: 698-701.

5. Bashour TT. Acute myocardial infarction resulting from amphetamine abuse: a spasm-thrombus interplay? Am Heart J 1994; 128: 1237-9.

6. Al Shehri Y. Multiple coronary thromboses in a young addict. J Saudi Heart Assoc 2016; 28: 180-4.

7. Wakman J, Tayler RN, Bodor GS, et al. Acute myocardial infarction associated with amphetamine use. Mayo Clin Proc 2001; 76: 323-6.

8. Won S, Hong RA, Shohet RV, Seto TB, Parikh NI. Methamphetamine-associated cardiomyopathy. Clin Cardio 2013; 36: 737-42.

9. Furst SR, Fallon SP, Reznik GN, Shah PK. Myocardial infarction after inhalation of methamphetamine. N Engl J Med 1990; 323: 1147-8.

10. El Menyar AA. Drug-induced myocardial infarction secondary to coronary artery spasm in teenagers and young adults. J Postgrad Med 2006; 52: 51-6.

11. Chen JP. Methamphetamine-associated acute myocardial infarction and cardiogenic shock with normal coronary arteries: refractory global coronary microvascular spasm. J Invasive Cardiol 2007; 19: E89-92. 